\title{
Fossile Mollusken in Kalksinterbildungen (Dauchen) am Lech-Ufer östlich von Hurlach (nördlich Landsberg/Lech)
}

\author{
JiRí KOVANDA *)
}

\author{
Pleistocene, tufa, sections, molluscs, \\ faunal lists, ecosystems, paleoclimatology, paleoenvironment \\ Bavaria, Alpenvorland
}

\begin{abstract}
Kurzfassung: Von Hurlach nördlich Landsberg a. Lech im Nördlichen Alpenvorland werden in ihrer Ausbildung stark variierende Sinterbildungen im Detail beschrieben und durch Fotos dokumentiert. Aus allen fossilführenden Schichten wurden die Mollusken bestimmt, aus den Kalkabsätzen ebenso wie aus den unterlagernden Flußablagerungen. Die paläoökologische Deutung der reich entwickelten Molluskengemeinschaften erlaubt Aussagen über das Sedimentationsmilieu und über das Biotop.
\end{abstract}

Als Bildungszeit kommt eine ausgeprägte Warmzeit in Betracht, höchstwahrscheinlich das letzte Interglazial. Die Molluskenarten lassen auf einen geschlossenen Laubmischwald mit feuchtem Charakter als Lebensraum schließen.

[Fossil Molluscs in Calcareous Freshwater Sediments in the Banks of the Lech East of Hurlach (North of Landsberg/Lech)]

Abstract: Near Hurlach in the Northern Alpine Foreland there are a wide variety of calcareous freshwater deposits. They are described in detail and illustrated with photos.

There is a list of the molluscs found in all fossil-bearing strata, both in the calcareous deposits and in the fluviatile sediments. The paleo-ecological interpretation of the richly developed molluscan fauna allows certain conclusions to be drawn about the biotop.

The time of sedimentation was a warm period, most probably the last interglacial period. The species of molluscs indicate that the biosphere was a coherent mixed foliaceous forest with a humid character.

\section{Einleitung}

Den am Lech östlich Hurlach gelegenen Fundort habe ich gemeinsam mit H. JeRZ, München, im Mai 1982

*) Anschrift des Autors: Dr. J. Kovanda, Geologische Zentralanştalt (ƯUUG), Malostranské nám. 19, CS - 11821 Praha 1, CSSR besucht. Das durch Flußerosion freigelegte Profil am linken Lechufer (Prallhang) befindet sich zwischen der 18. und 19. Lechstaustufe in einem Auwaldgebiet (s. Abb. 1). Das bogenförmige Uferprofil wies damals eine Höhe von 4 bis $4,5 \mathrm{~m}$ über dem Flußwasserspiegel auf; die Länge des untersuchten Profils betrug $32 \mathrm{~m}$.

Das Profil setzt sich aus.fünf lithofaziell verschiedenen Sedimenttypen zusammen: An der Basis sind es Terrassenschotter, die von schluffigen Mergeln überdeckt sind. Den Hauptteil der Schichtenfolge bilden verschiedene Kalksinterbildungen (Dauch- und Traver-

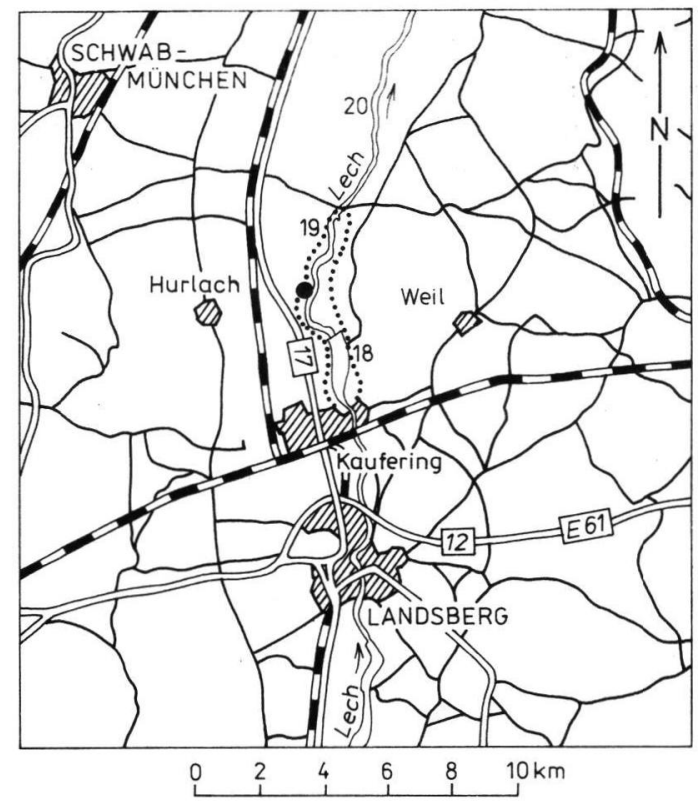

Abb. 1: Lageskizze des Kalksintervorkommens Hurlach nördlich Landsberg a. Lech. Die Lage des Uferprofils ist mit einem schwarzen Punkt bezeichnet. 
tintypen; vgl. KOvANDA 1983), die im unteren Abschnitt eine durchlaufend verfolgbare, geringmächtige Lage eines völlig zersetzten Torfes führen. Darüber folgen bis zur Oberfläche Flußschotter, die vereinzelte, abgerollte Travertinblöcke enthalten. - Vgl. auch JERZ \& MANGELSDORF, in diesem Band).

\section{Beschreibung der Schichtenfolge des Uferprofils}

Die Aufschlußwand am Lechufer wurde in $\mathrm{z}$ e h $\mathrm{n}$ Profilen aufgenommen (Lage der Profile siehe Abb. 2: Ziffern über der Profilskizze). Das basale Glied der Schichtenfolge (Probe Nr. 1, s. Abb. 2: Ziffer mit Kreis umrandet) - sandige Flußkiese, unten rostfarben, nach oben zu grau - ist nur im linken (südlichen) Aufschlußteil sichtbar (Profile Nr. 9 und 10). Die Obergrenze dieser Schotter lag nur 1 Meter über dem Flußwasserspiegel. Den Schottern ist ein grünlich- und bläulichgrauer, unten schwach toniger (Probe Nr. $2 \mathrm{a}$ in Abb. 2), im mittleren Teil mehr sandigschluffiger Flußmergel (Probe Nr. 2b) semidiskordant aufgelagert. Der obere Teil dieser Mergel (Probe Nr. 2c) enthält bereits einen gewissen Anteil an sandigen Dauchinkrustationen, der den Beginn der biochemischen Kalkablagerung am Fundort belegt.

Im rechten Aufschlußteil (Profile Nr. 1 bis 4 in Abb. 2 ) ist die Obergrenze der Flußmergel unregelmäßiger; die Unebenheiten ihrer Oberfläche werden durch eine dünne Lage grobsandiger Inkrustationen überdeckt.

Zum Hangenden folgt - wieder diskordant - ein Horizont mit unten grobkörnigen, zum Teil umgelagerten (Probe Nr. 3a, s. Abb. 2), nach oben mehr feinkörnigen, schluffig-sandigen Dauchen (Probe Nr. 3b). In den Profilen Nr. 5, 6, 7 und 9 (vgl. Abb. 2) ist diese Lage als unregelmäßiger, bis $10 \mathrm{~cm}$ mächtiger Horizont entwickelt, der aus stellenweise schwach humosen, anderswo grobsandigen, ebenfalls zum Teil umgelagerten Dauchen aufgebaut ist; diese füllen in Profil Nr. 5 auch eine Krotowine in den unterlagernden schluffigen Mergeln aus.

Ein ausgeprägter, an der ganzen Uferprofilwand durchgehender Leithorizont ist eine schwarze, 0,5 bis $3 \mathrm{~cm}$ mächtige Lage völlig zersetzter und zusammengepreßter Torfe, die in den Profilen 1 bis 4 in Abb. 2 auf einem schwach violetten, dunkelbraunen, stark humosen Mergelhorizont (Probe Nr. 4 in Abb. 2) entwickelt sind. Dieser Horizont liegt auf einer unebenen Unterlage. An einigen Stellen bildet er infolge des Drucks der überlagernden Karbonatgesteine eingestülpte Falten (vgl. Abb. 5).

An diesem Torf wurden absolute Altersbestimmungen mit der Radiokohlenstoff-Methode durchgeführt. Zwei Datierungen ergaben ${ }^{14} \mathrm{C}$-Alter zwischen 30000 und 35000 Jahren B.P. (Prof. Dr. M. A. GEYH, Niedersächs. Landesamt $f$. Bodenforsch., Hannover). Im Hangenden folgt in den Profilen Nr. 1 bis 4 in Abb. 2 eine durchgehende Lage (Probe Nr. $5 \mathrm{a}$ in Abb. 2) mit hellockerfarbenen bis gelblichen, feinund mittelsandigen, an der Basis schwach graugefärbten, schwach humosen, mergeligen Dauchen. In Profil Nr. 1 wird sie noch durch eine Linse aus ockerfarbenem, moosartigem, leicht zerfallendem Dauch unterlagert. In der Mitte der Aufschlußwand ist der erwähnte Horizont als Lage mit grauen bis ockerfarbenen, schwach mergeligen, sandigen Dauchen mit humosen Schlieren (Probe Nr. 5b, s. Abb. 2) entwickelt. Im linken Aufschlußteil (Profile Nr. 7 bis 10 in Abb. 2) wird er wieder durch ocker- und rostfarbene, grobkörnige, sandige Dauche (Probe Nr. 5c) faziell vertreten, die jedoch mit kleinbröckelig zer-

SSW

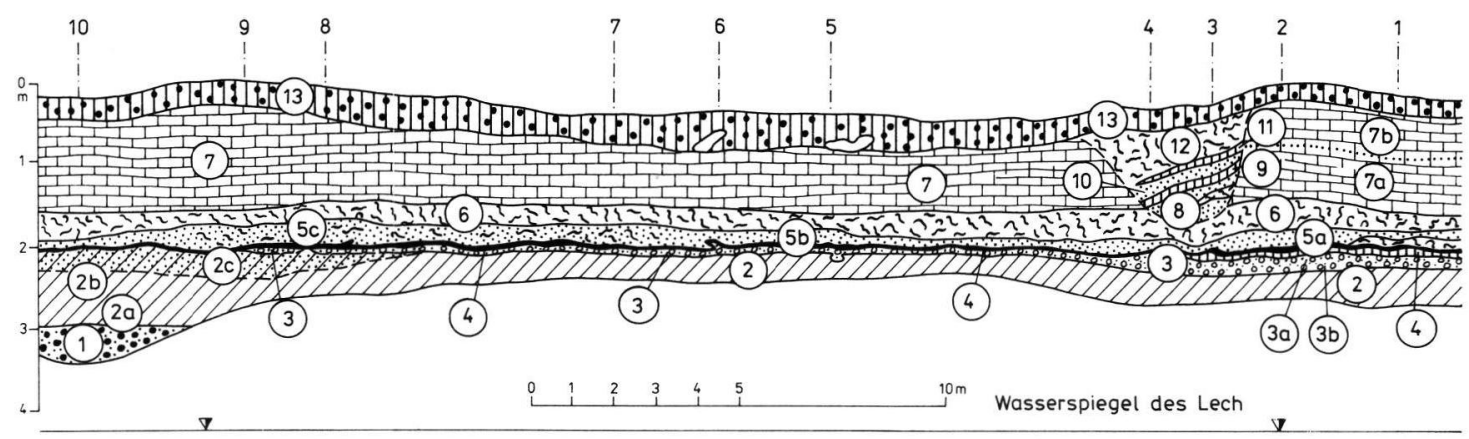

Abb. 2: Schematische Darstellung des Uferprofils am Lech östlich Hurlach.

Die über dem Profil angegebenen Ziffern 1 bis 10 bezeichnen die vermessenen Profile, die in den Kreisen eingeschlossenen Ziffern 1 bis 13 die Entnahmestellen der Proben. - Beschreibung der Schichtenfolge siehe im Text. 
fallenden, eine Zwischenmasse aus sandigen Dauchen enthaltenden, festeren Travertinen abwechseln bzw. gemeinsam vorkommen.

Die nächst höhere Lage (Probe Nr. 6 in Abb. 2) besteht aus hellockerfarbenen bis goldgelben, meist zerfallenden, moos- und algenartigen Strukturdauchen, die stellenweise zahlreiche Algenknollen aufweisen. In Profil Nr. 7 in Abb. 2 befindet sich in dieser Lage eine $25 \times 15 \times 8 \mathrm{~cm}$ große Kaverne, deren Wände mit sekundärem Sinterkalk überzogen sind. Daraus stammt auch ein inkrustiertes Riedgrasbüschel. In Profil Nr. 8 sind an diese Lage rostbraune und schwarze Ausfällungen mit Fe- und Mn-Oxiden bzw. -Hydroxiden gebunden.

Die mächtigste und widerstandsfähigste Bank in der ganzen Schichtenfolge bilden horizontal gelagerte, dünn geschichtete, feste, poröse Dauche bis Travertine (Quellkalke sensu KovANDA 1971). Ihr unterer Teil ist ziemlich kompakt (Probe Nr. $7 \mathrm{a}$ in Abb. 2), der obere Teil zerfällt blockartig (Probe Nr. 7b). In diesen festen Schichten ist in den Profilen 3 und 4 eine kleine (?Erosions-) Rinne ausgebildet, die mit schräg gelagerten, etwas jüngeren Dauchen sowie mit zwei humosen initialen Rendzinaböden ausgefüllt ist. An der Basis dieser Ausfüllung liegen hellockerfarbene, sandige Dauche, in der Mitte mit einer Linse von Strukturdauchen und groben Inkrustationen (Probe Nr. 8). Darüber folgt eine Schicht mit einer wenig entwickelten, hellgrauen und bräunlichen, humosen Rendzina (Probe Nr. 9), die wiederum aus sandigen und schwach mergeligen Dauchen entwickelt ist. Sie wird von gelblichen, sandigen Dauchen überdeckt, mit einem Anteil an kleinbröckeligen Strukturdauchen (Probe Nr. 10). An ihrer Oberfläche ist eine weitere, schwach humose initiale Rendzina ausgebildet (Probe Nr. 11). Die oberste Schicht der Ausfüllung bilden blockartig zerfallende Moosdauche (Probe Nr. 12).

Das jüngste Glied des gesamten Profils bilden Flußschotter mit einem darauf entwickelten Rendzinaboden. In den Profilen 5 und 6 (s. Abb. 2) liegen darin mehrere Dezimeter große Blöcke kompakter, an der Oberfläche stark korrodierter und gerundeter Travertine.

\section{Mollusken der fossilführenden Schichten}

(Proben-Nr. vgl. Abb. 2)

Größere Orientierungsproben zur paläomalakologischen Auswertung wurden allen fossilführenden Horizonten des Profils, darunter auch den basalen sandigen Flußschottern, entnommen. Alle Proben wiesen ungefähr die gleiche Materialmenge auf (etwa 2 bis $2,5 \mathrm{~kg}$ ). Den vertikal bzw. horizontal lithofaziell wechselnden Schichten wurden getrennte, mit den Buchstaben a, b, c bezeichnete Proben entnommen. Sie wurden mit Hilfe eines Siebes von $0,5 \mathrm{~mm} \mathrm{Ma-}$ schenweite geschlämmt. Aus den sandigen Schottern wurde die Fraktion 0,5-4,0 mm ausgesiebt. Die Molluskengehäuse liegen häufig nur als Fragmente vor. Unversehrt erhalten geblieben sind nur ausnahmsweise winzige Schalen oder auch Individuen mit festeren Gehäusen, wie z.B. der Gattung Valvata und zum Teil Gehäuse der Art Lymnaea peregra (MÜLL.) in der Lage der Probe Nr. 5c. Ausgeschlämmt wurden fossile Reste von 55 Molluskenarten (siehe Tab. 1). Als reichste Mollusken-führende Lage erwies sich der torfige Horizont (Probe Nr. 4) bzw. sein Liegendes, das vornehmlich aus verschiedenkörnigen Dauchsanden (Probe Nr. 3) besteht. Als ähnlich reichhaltig darf der Humushorizont einer initialen Rendzina (Probe Nr. 9) angesehen werden, der ungefähr in der Mitte der aufgefüllten (?Erosions-) Rinne in den Kalksintern angetroffen worden ist. Malakozoologisch am ärmsten ist die basale Dauchschicht in der genannten Rinnenfüllung (Probe Nr. 8). - Vgl. Tab. 1.

In der ausgeschlämmten Molluskenfauna sind insbesondere zwei ausgeprägte Waldbewohner bemerkenswert, nämlich Aegopis verticillus (LAM.) und Pagodulina pagodula (DESM.). Die erstere ist durch mehrere typische Bruchstücke in der Torfschicht s. 1. (Probe Nr. 4) sowie in den darunter liegenden Dauchsanden (Probe Nr. 3) vertreten. Die letztere fand sich im Hangenden der erwähnten Torfschicht (vereinzelt auch in der Probe Nr. 5), außerdem sehr häufig in der Mitte der oben genannten Rinnenfüllung (Probe Nr. 9) und schließlich vereinzelt in den hangenden Dauchen (Probe Nr. 10). Die Vorkommen der beiden erwähnten Arten befinden sich im Profil also in zeitlich aufeinanderfolgenden Ablagerungen. Bemerkenswert sind weiter auch die Vorkommen der Arten Helicodonta obvoluta (MÜLL.), Monachoides incarnata (MÜll.), Semilimax semilimax (Fér.) und Discus rotundatus (MÜLL.), die den gesamten unteren Profilteil (Proben Nr. 1 bis 4) zusammenhängend durchlaufen, was ähnlich auch für Discus perspectivus (MEG. v. MüHL.) gilt, welche jedoch in den sandigen Schottern sowie in den Schluffmergeln (Proben Nr. 1 und 2) vorläufig noch nicht festgestellt worden ist.

Die Artenspektren der Faunen im unteren Profilteil (Proben Nr. 1 bis 5) und die Dauchausfüllung der Rinne (Proben Nr. 8 bis 10) sind fast gleich. Allerdings ist die Artenzahl in den Proben der Rinnenfüllung etwas kleiner. Eine einzige Ausnahme stellt hier die oben erwähnte Art Pagodulina pagodula (DESM.) dar, die erst in den jüngeren Kalksinterbildungen zum Vorschein kommt. Die auffällig größere Arten-(und Individuen-) Zahl in der Lage der Probe 9 gegenüber der Liegendschicht (Probe Nr. 8) sowie der 


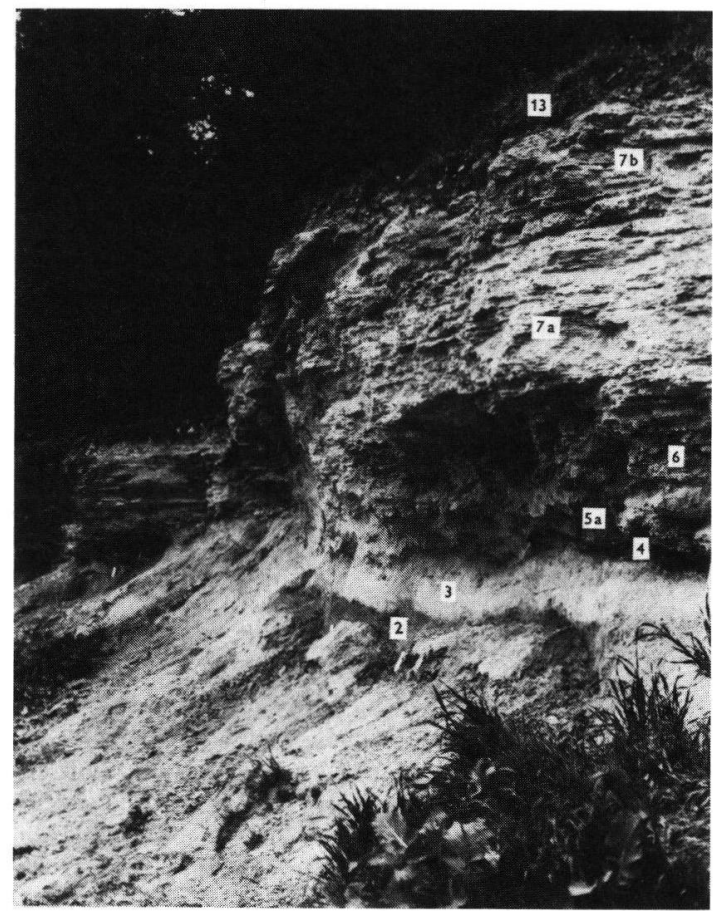

Abb. 3: Seitenansicht des Profils der Travertinund Dauchlagerstätte am linken Lechufer.

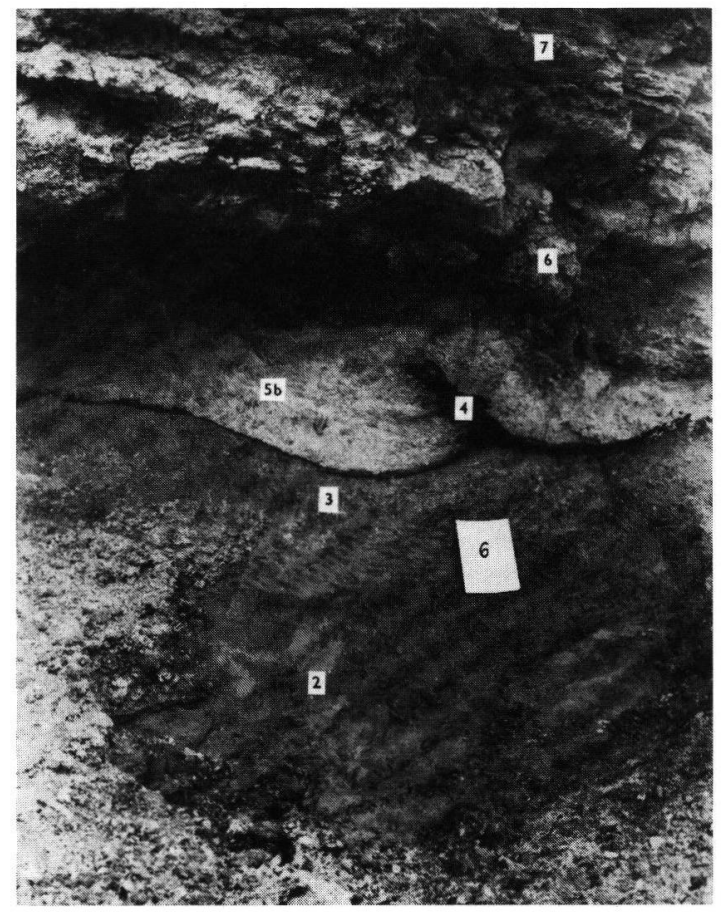

Abb. 5: Detailansicht des mittleren Teiles des Uferprofils (Profil 6 in Abb. 2).

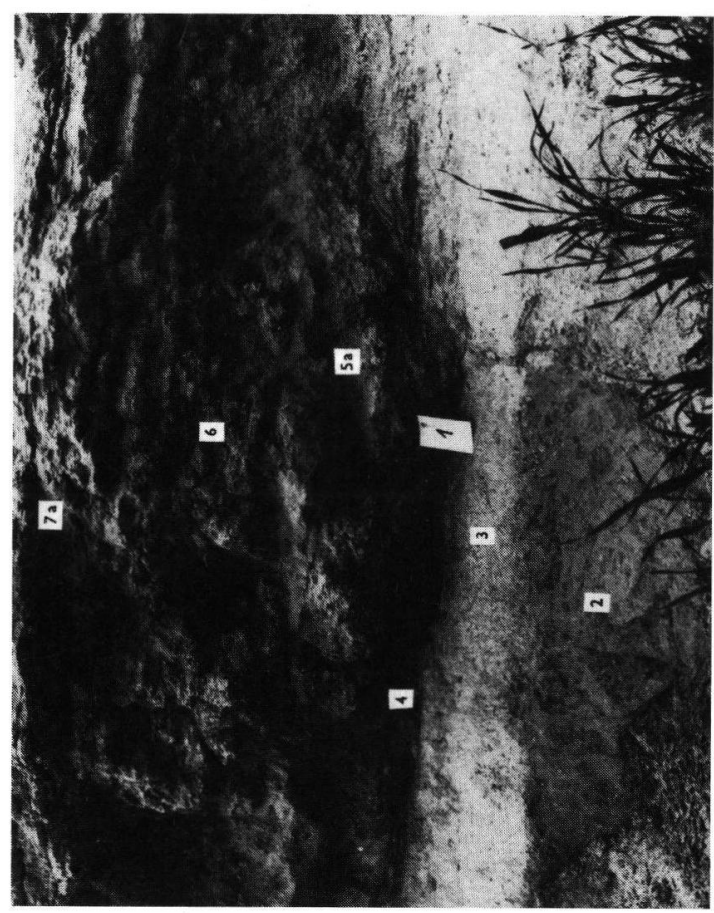

Abb. 4: Detailansicht des rechten Teiles des Uferprofils (Profil 1 in Abb. 2).

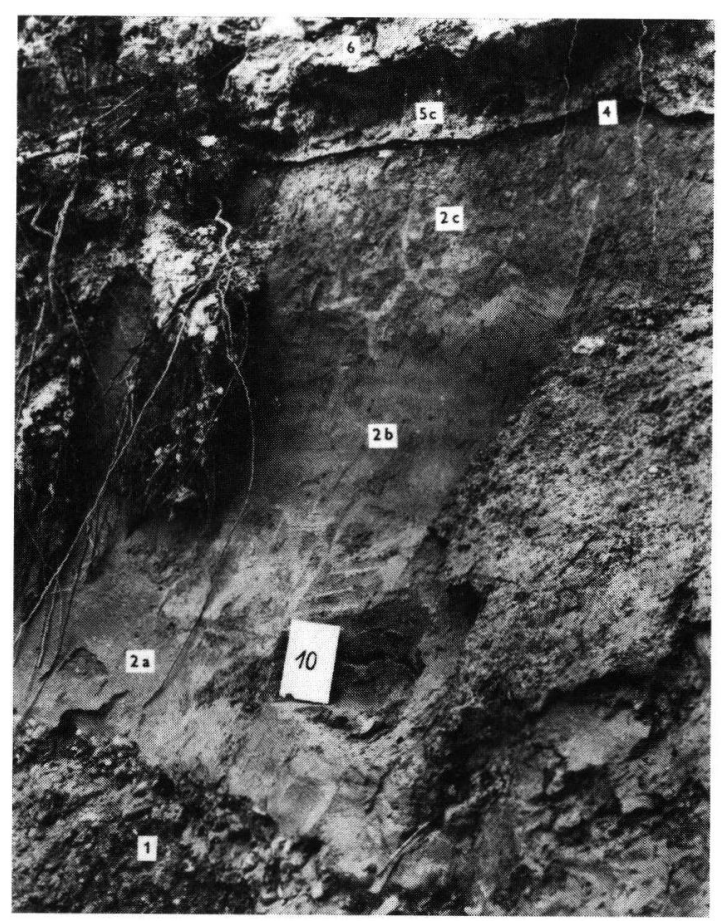

Abb. 6: Detailansicht des linken Teiles des Uferprofils (Profil 10 in Abb. 2). 
Hangendschicht (Probe Nr. 10) hängt mit den Entstehungsbedingungen der genannten Horizonte zusammen: Während die Sand- und Strukturdauche sehr schnell zur Ablagerung kamen, erfolgte die Sedimentation der humosen, mergeligen Lage (Probe Nr. 9) unvergleichlich langsamer; sie enthält deshalb einen größeren Reichtum an Mollusken (vgl. KovANDA 1971; LOŽEK 1973, 1976, 1982).

\section{Paläoökologische Deutung}

Die aus dem Profil gewonnene Molluskenfauna stellt eine Thanatozönose der ökologischen Gruppen Nr. 1 bis 3 und 7 bis 10 sensu LOŽEK (1964) dar (s. Tab. 1). Die Arten der ökologischen Gruppen Nr. 4, 5 und 6, d. h. Steppenbewohner, Bewohner offener Standorte und xerotherme Arten fehlen völlig. Bemerkenswert ist, daß von der basalen Lage der sandigen Flußschotter zum Hangenden im Profil durchlaufend die Waldbewohner zu verfolgen sind. Dies ist ein unerwarteter Befund, denn in ähnlichen Aufschlüssen in Mitteleuropa kommen in Schottern und Mergeln im Liegenden von Dauchablagerungen Faunen der ausklingenden Glazialzeit bzw. die sog. Übergangsfaunen (sensu LOŽEK 1964) vor, die eben durch Arten der ökologischen Gruppen Nr. 4 und 5 vertreten sind.

In der ermittelten Molluskenfauna überwiegen deutlich terrestrische Arten ( $72 \%$ ) gegenüber solchen der Gewässer und Sümpfe (28\%), was für Dauch- und Travertinablagerungen typisch ist (KovANDA 1971). Die größte Häufigkeit von Wasserarten ist an die Torflage (Probe Nr. 4), an ihr unmittelbares Liegendes (Probe Nr. 3) sowie ihr Hangendes (Probe Nr. 5) gebunden. Ungeachtet dessen wird der subaquatische Ursprung aller Horizonte durch Wasserschnecken belegt, die das Profil praktisch durchlaufend in seiner gesamten Mächtigkeit kennzeichnen.

Die ökologischen Ansprüche der gefundenen Wasserschnecken sind im ganzen gesehen gleich. Sie belegen seichte, stehende bzw. mäßig durchströmte, z.T. auch periodisch durch eine Vegetationsdecke weitgehend zugewachsene Wasserbiotope vom Typ der Altwasserarme und Sümpfe in den Wiesen- bzw. Sumpfwiesenbiotopen. In der terrestrischen Komponente überwiegen völlig „Waldarten” s. 1.: (ökologische Gruppe Nr. 1), die zusammen mit „Waldsteppenarten" bzw. Arten der lichten Haine (ökologische Gruppe Nr. 2) und mit einer Art des Sumpfwaldes (ökologische Gruppe Nr. 3 sensu LOŽEK 1964) insgesamt etwa die Hälfte der Artenzahl aus allen ermittelten ökologischen Gruppen ausmachen, wenn die erwähnten Wasserbiotope (ökologische Gruppe Nr. 10) und Sumpfbiotope (ökologische Gruppe Nr. 9) in die Berechnung einbezogen werden. Es handelt sich also um eine reich entwickelte Molluskengemeinschaft, durch die belegt ist, daß am Fundort zur Zeit der Ablagerung der beschriebenen Schichtenfolge ein zusammenhängender Laubmischwald von feuchtem Charakter mit begrenzten Enklaven von lichtem Wald- und Waldsteppentyp existierte. Durch die hier vorkommenden Mollusken der ökologischen Gruppe Nr. 7 (ökologisch indifferente Arten) und Nr. 8 (feuchtigkeitsliebende Arten) wird der vorliegende Artenreichtum nur ergänzt und erweitert.

$\mathrm{Z} u \mathrm{~s}$ a m m e $\mathrm{n}$ a s s e $\mathrm{nd}$ darf man folgendes ableiten: Die artenmäßig einheitliche Vergesellschaftung fossiler Mollusken schwankt zwar mengenmäßig, zeigt aber einen im Prinzip gleichbleibenden Charakter der Ablagerungsbedingungen im Entstehungszeitraum an. Dies bedeutet, daß die ganze Ablagerungsfolge in einem hinsichtlich Klima und Biotop verhältnismäßig beständigen Milieu, ohne ausgeprägte paläoklimatische Wandlungen und ohne Biotopänderungen entstanden ist. Die Ablösung der minerogenen Ablagerung in der Profilbasis durch die biogenchemogenen Karbonate wurde durch reichlich Calciumbicarbonat enthaltendes Quellwasser verursacht. Die geringmächtige torfige Lage deutet auf eine kurze Unterbrechung des Quellwasserzuflusses hin, während welcher die Karbonat-Ausfällungen am Fundort fast aufhörten, weshalb eine gewisse Zeit lang hier vornehmlich nur organodetritische Bildungen entstanden.

Auch die zur Bildung der gesamten Ablagerungen notwendige Zeit war unter Berücksichtigung der oben angeführten Befunde verhältnismäßig sehr kurz, was durch fast analoge Artenspektren der Molluskenfaunen (MSS - s. Abb. 7) bezeugt wird. Nur im Hangenden der Torflage Nr. 4 (d. h. im jüngeren Abschnitt der Schichtenfolge mit den Proben Nr. 5 bis 10) ist eine Abnahme der Waldbewohner zugunsten der relativen Zunahme des Anteils anderer ökologischer Gruppen zu beobachten. In paläoökologischer und somit auch in paläoklimatischer Hinsicht handelt es sich demnach um die Zeit einer maximalen Entwicklung sowie eines nachfolgenden teilweisen Rückganges geschlossener Laubmischwälder.

\section{Stratigraphische Deutung}

Aus der im Profil Hurlach bestimmten Molluskenfauna kann eine Warmzeit erster Ordnung - wie das Holozän oder ein Interglazial - , und zwar ein Klimaoptimum und dessen allmähliche Abschwächung abgeleitet werden.

Mit der Sinterkalkbildung, die ihr Maximum in den erwähnten Zeitperioden erreicht, steht die von uns 
Tab. 1: Verzeichnis fossiler Mollusken vom Fundort Hurlach. Die Ziffern 1 bis 3 und 7 bis 10 bezeichnen die ökologischen Gruppen nach LOŽEK (1964): 1-Waldbiotope s. 1.; 2 - lichte Haine und Waldsteppen; 3 - Auwald; 7 - ökologisch indifferente Arten; 8 - feuchte Standorte;

9-Sümpfe und Gewässerufer; 10-Wasserbiotope. !! - Arten der Höhepunkte der quartären Klimaoptima; ! - Arten der warmen und feuchten Klimaperioden.

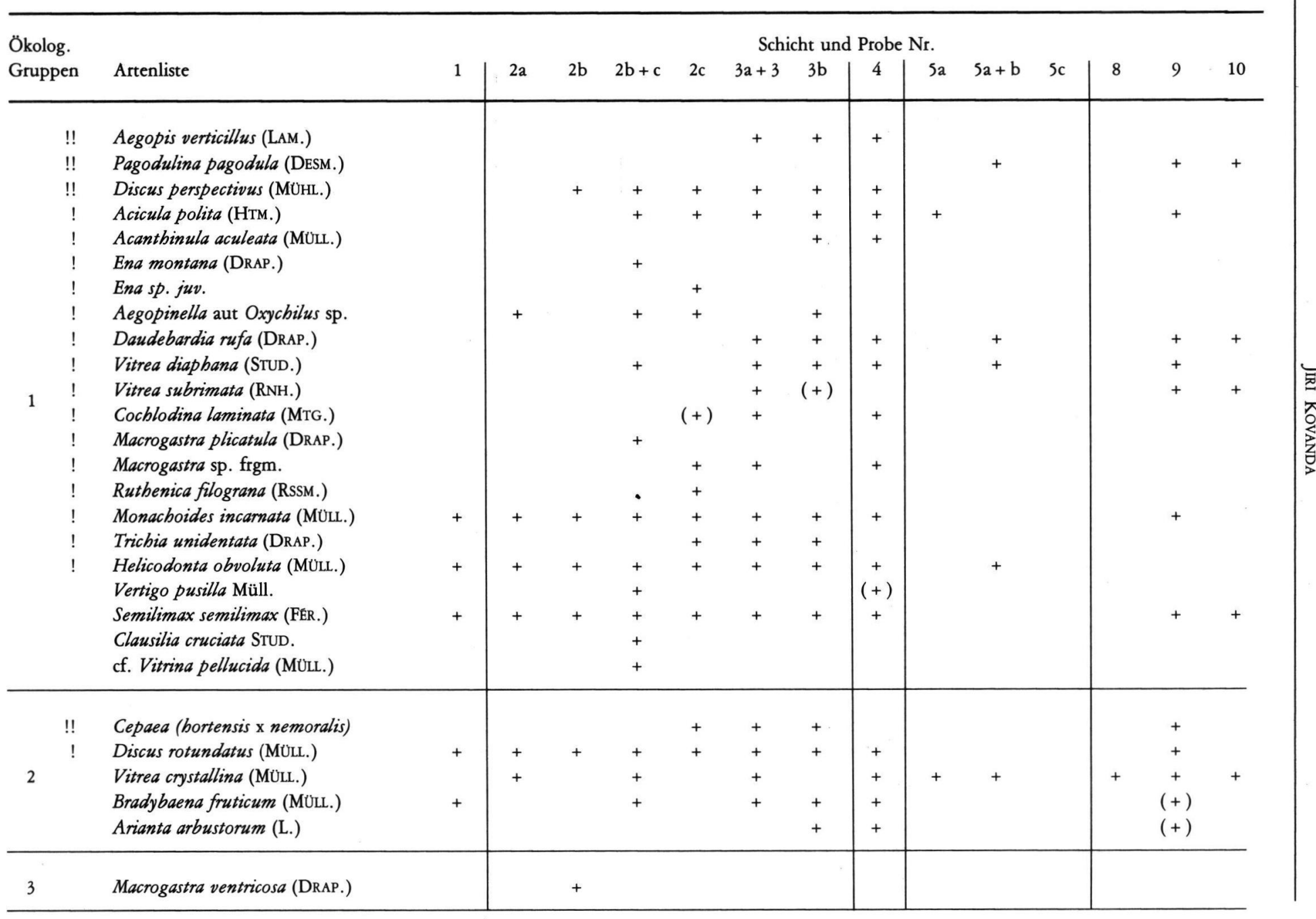




\begin{tabular}{|c|c|c|c|c|c|c|c|c|c|c|c|c|c|c|c|c|}
\hline 7 & & $\begin{array}{l}\text { Cocblicopa lubrica (MUULL.) } \\
\text { Punctum pygmaeum (DRAP.) } \\
\text { Perpolita bammonis (STRÖ) } \\
\text { Vitrea contracta (WEST.) } \\
\text { Euconulus fulvus (MÜLI.) } \\
\text { Clausilia dubia DRAP. } \\
\text { Trichia cf. sericea (DRAP.) } \\
\text { Tricbia cf. bispida (L.) }\end{array}$ & & + & & + & + & $\begin{array}{l}+ \\
+\end{array}$ & $\begin{array}{l}+ \\
+ \\
+ \\
+ \\
+\end{array}$ & $\begin{array}{c}(+) \\
+\end{array}$ & + & $\begin{array}{c}+ \\
(+)\end{array}$ & + & & $\begin{array}{l}+ \\
+ \\
+ \\
+ \\
+\end{array}$ & $\begin{array}{l}+ \\
+ \\
+\end{array}$ \\
\hline 8 & $!$ & $\begin{array}{l}\text { Carychium tridentatum (RISSO) } \\
\text { Vertigo angustior JEFFR. } \\
\text { Vertigo substriata (JEFF.) } \\
\text { Succinea oblonga DRAP. }\end{array}$ & & + & & + & & + & + & + & + & + & & & $\begin{array}{l}+ \\
+ \\
+\end{array}$ & + \\
\hline 9 & & $\begin{array}{l}\text { Carychium minimum MÜLL. } \\
\text { Vertigo antivertigo (DRAP.) } \\
\text { Succinea putris (L.) } \\
\text { Oxyloma elegans (RISSO) } \\
\text { Succinea x Oxyloma juv. } \\
\text { Zonitoides nitidus (MÜLL.) }\end{array}$ & $i$ & & & & & $\begin{array}{l}+ \\
+ \\
+\end{array}$ & + & $\begin{array}{l}+ \\
+ \\
+\end{array}$ & $\begin{array}{l}+ \\
+\end{array}$ & + & + & & $\begin{array}{l}+ \\
+ \\
+\end{array}$ & + \\
\hline 10 & & $\begin{array}{l}\text { Lartetia sp. } \\
\text { Valvata cristata MUULL. } \\
\text { Valvata piscinalis (MÜLL.) } \\
\text { Valvata pulcbella (STUD.) } \\
\text { Lymnaea truncatula (MÜL.) } \\
\text { Lymnaea peregra (MÜLL.) } \\
\text { Lymnaea sp. frgm. } \\
\text { Planorbis planorbis (L.) } \\
\text { Anisus leucostomus (MILET) } \\
\text { Armiger crista (L.) } \\
\text { Pisidium sp. }\end{array}$ & + & & + & $\begin{array}{l}+ \\
+ \\
+\end{array}$ & $\begin{array}{l}+ \\
+ \\
+\end{array}$ & $\begin{array}{l}+ \\
+ \\
+ \\
+ \\
+ \\
+ \\
+ \\
+ \\
+\end{array}$ & $\begin{array}{l}+ \\
+ \\
+ \\
+ \\
+ \\
+ \\
+\end{array}$ & $\begin{array}{l}+ \\
+ \\
+ \\
+ \\
+ \\
+\end{array}$ & $\begin{array}{l}+ \\
+ \\
+ \\
+ \\
+ \\
+ \\
+\end{array}$ & + & $\begin{array}{l}+ \\
+ \\
+\end{array}$ & $\begin{array}{l}+ \\
+ \\
+\end{array}$ & $\begin{array}{l}+ \\
+ \\
+ \\
+ \\
+ \\
+\end{array}$ & $\begin{array}{l}. \\
+ \\
+\end{array}$ \\
\hline$?$ & & $\begin{array}{l}\text { Vertigo x Columella frgm. } \\
\text { Limacideae sp. div. } \\
\text { Clausiliideae sp. frgm. + juv. } \\
\text { Trichia sp. frgm. } \\
\text { Helicideae sp. }\end{array}$ & & + & + & & & + & + & $\begin{array}{l}+ \\
+\end{array}$ & $\begin{array}{l}+ \\
+\end{array}$ & + & + & + & + & + \\
\hline
\end{tabular}


entworfene klimatisch-ökologische Einstufung der Karbonat-Schichtenfolge im Einklang. Anders sieht es - wie bereits oben erwähnt - mit dem basalen sandigen Flußschotter und Auemergel aus. Das Auftreten einer Waldfauna in diesen minerogenen Schichten bezeugt, daß es sich nicht etwa um fluvioglaziale Sedimente, sondern um die oberste Lage eines bereits in einer Warmzeit umgelagerten Schotters handelt.

Es bleibt noch die Frage zu lösen, ob die beschriebenen Sedimente ein postglaziales (holozänes) oder ein interglaziales Alter, d. h. höchstwahrscheinlich letztinterglaziales Alter aufweisen. Zu einer endgültigen Entscheidung darüber kann die Paläomalakoanalyse nicht allein ausschlaggebend sein, da keine vergleichbaren, eingehend bearbeiteten und statistisch ausgewerteten Profile aus der weiteren Umgebung unseres Fundortes zur Verfügung stehen, wie dies z. B. in der Tschechoslowakei der Fall ist. Deshalb kann die Fluktuation einiger Molluskenarten in diesem Gebiet nicht unmittelbar beurteilt werden, wofür in erster Linie die beiden oben erwähnten ,Waldarten”, nämlich Aegopis verticillus (LAM.) und Pagodulina pagodula (DESM.) in Betracht kämen.

Die balkanisch-ostalpine Art Aegopis verticillus (LAM.), die auch in der Tschechoslowakei verbreitet ist, lebt in Bayern in der Umgebung von Berchtesgaden (Schellenberg) und Passau sowie außerdem offensichtlich künstlich verpflanzt - auch am Lech bei Landsberg, d. h. nur wenige Kilometer flußaufwärts südlich von Hurlach (JAECKEL 1962). Es handelt sich um eine über fast ganz Mitteleuropa verbreitete hochinterglaziale Leitart. In der Tschechoslowakei wurde sie kürzlich von V. LOŽEK (nicht publ.) auch im Holozän des Mährisichen Karstes nördlich von Brno festgestellt, wo sie allerdings auch rezent vorkommt.

Die alpin-osteuropäische Art Pagodulina pagodula (DESM.) lebt in Bayern (ähnlich wie die zuvor angeführte Art) in der weiteren Umgebung von Berchtesgaden und an den Ufern entlang des Inns (JAECKEL 1962). Sie pflegt ebenfalls ein Bestandteil der hochinterglazialen Faunen zu sein. Aus dem Holozän in Mitteleuropa ist sie vermutlich nicht bekannt.

Auch Discus perspectivus (MEG. v. MÜHL.), eine ostalpin-balkanische Art, ist bemerkenswert. Rezent ist sie in Bayern nördlich Kufstein, in der Umgebung von Neuburg a. Inn, von hier bis nach Burghausen an der Salzach, dann in der weiteren Umgebung von Berchtesgaden, ferner bei Passau, an der Isar südlich von München sowie verstreut im Allgäu, z. B. bei Kaufbeuren a. d. Wertach, das dem Fundort Hurlach am nächsten liegt. Schließlich ist diese Art auch im Raum Nürnberg verbreitet (JAECKEL 1962). Außer in
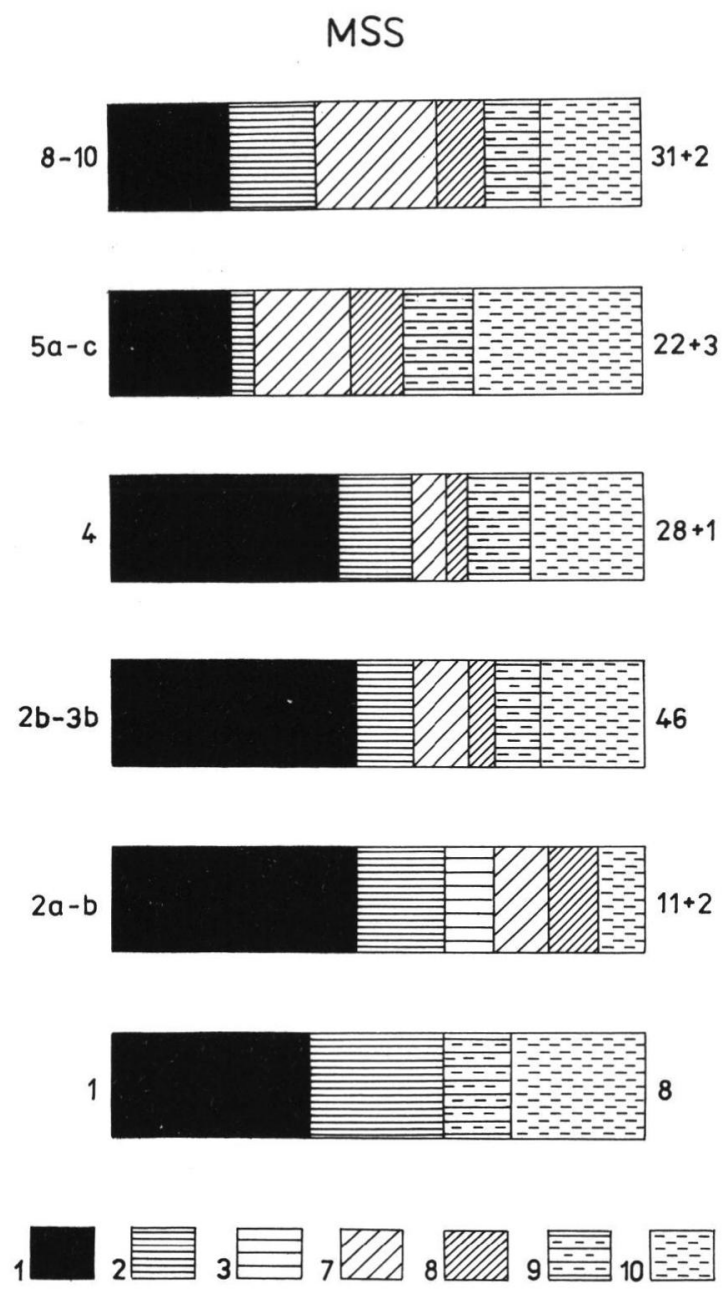

Abb. 7: Molluskenspektren der Arten (MSS) vom Fundort Hurlach, konstruiert nach der Methode von LOZEK (1964). Die Ziffern 1 bis 3 und 7 bis 10 bezeichnen ökologische Gruppen (siehe Erläuterung zur Tabelle 1).

Links von den Spektren sind die Nummern der ausgewerteten Proben, rechts die ermittelten Artenzahlen angeführt. Die Breite eines Spektrums entspricht $100 \%$.

interglazialen kommt sie auch in holozänen Ablagerungen vor.

Die übrigen Arten stellen einen ziemlich häufigen Bestandteil günstiger rezenter Biozönosen in der näheren Umgebung von Hurlach dar.

Für holoz ä nes Alter der Dauchbildungen könnten zunächst folgende Tatsachen sprechen:

(a) Die Lage des Profils, das sich in der Talaue mit der Oberkante der Schichtenfolge nur etwa $4 \mathrm{~m}$ über dem Wasserspiegel des Lechs befindet. 
(b) Das ziemlich frische Aussehen der Dauchschichten, dazu mit einer Torflage, die in interglazialen Sedimenten dieses Typs nur sehr selten vorkommt, sowie zwei Rendzinaböden in einem Anfangsstadium ihrer Entwicklung.

(c) . Ein im großen und ganzen mit rezenten Molluskengemeinschaften in der näheren Umgebung übereinstimmender Charakter der Molluskenfauna - mit Ausnahme der oben angeführten Arten Aegopis verticillus (LAM.), Pagodulina pagodula (DESM.), evtl. auch Discus perspectivus (MEG. v. MÜHL.).

Für das Klimaoptimum höchstwahrscheinlich des letzten Interglazials sprechen demgegenüber folgende Befunde:

(a) Das Vorkommen der oben erwähnten hochinterglazialen Leitarten Aegopis verticillus (LAM.) und Pagodulina pagodula (DESM.).

(b) Der Umstand, daß die Dauche und Travertine seit langem nicht mehr mit Quellen in Verbindung stehen, aus denen sie ausgefällt worden sind.

(c) Die Radiokohlenstoff-Datierung der Torflage (Probe Nr. 4) mit einem ${ }^{14} \mathrm{C}$-Alter von mindestens 35000 Jahren schließt das holozäne Alter der Ablagerungen aus (vgl. JERZ \& MANGELSDORF 1989, i. ds. Band).

$\mathrm{Z}$ u s a m m e $\mathrm{n} f$ a s s e $\mathrm{nd}$ kann festgestellt werden, daß die Sinterkalke am Lechufer östlich Hurlach ein reiches paläomalakozoologisches Material aufweisen. Aus der paläoökologischen Analyse wird [die Folgerung] abgeleitet, daß sowohl die Süßwasserkalke als auch die unterlagernde minerogene Schichtenserie zur Zeit des Klimaoptimums höchstwahrscheinlich. des letzten Interglazials (Riß/Würm) und zu Beginn seiner allmählichen Abschwächung zum Absatz kamen. Es handelt sich um ein sehr bedeutendes Profil, dessen Untersuchung zur Kenntnis der natür- lichen Umweltbedingungen an einer Stelle in Südbayern für den genannten Zeitraum beigetragen und einen Einblick in den Reichtum und die örtliche Vielfalt fossiler Molluskenfaunen geboten hat. Sie sind den hiesigen rezenten Molluskengemeinschaften ziemlich ähnlich.

\section{Schriftenverzeichnis}

JAECKEL, S. G. H. (1962): Ergänzungen und Berichtigungen zum rezenten und quartären Vorkommen der mitteleuropäischen Mollusken. - In: Die Tierwelt Mitteleuropas, Bd. 2, Ergänzung. - Liefg. 1: 25-260; Leipzig.

JeRZ, H. \& MANGeLSDORF, J. (1989): Die interglazialen Kalksinterbildungen bei Hurlach nördlich Landsberg am Lech. - Eiszeitalter u. Gegenwart, 39: 29-32; Hannover.

Kovanda, J. (1971): Kvartérní vápence Československa. Quartärkalke in der Tschechoslowakei. - Sbor. geol. Vèd, R. A, Antropozoikum, 7: 7-236; Praha.

- (1983): Holozäne Süßwasserkalke und ihre Bedeutung für die Gliederung der Flußablagerungen in der Tschechoslowakei. - Geol. Jb., A 71: 285-289; Hannover.

LOŽEK, V. (1964): Quartärmollusken der Tschechoslowakei. - Rozpr. Ústrv. Úst. Geol., 31: 1-374; Praha.

- (1973): Príroda ve čtvrtohorách. - 372 S.; Praha (Academia Praha).

- (1976): Klimaabhängige Zyklen der Sedimentation und Bodenbildung während des Quartärs im Lichte malakozoologischer Untersuchungen. - Rozpr. Čs. Akad. Věd, R. matem.-prúr. Věd, 86 (8): 1—97; Praha.

- (1982): Faunengeschichtliche Grundlinien zur spätund nacheiszeitlichen Entwicklung der Molluskenbestände in Mitteleuropa. - Ropr. C's. Akad. Vèd, $\breve{R}$. matem.-prír. Věd, 92 (4): 1-106; Praha.

Manuskript eingegangen am 13.12. 1988. 